\title{
Evaluation des facteurs d'ambiances physiques de travail à la Compagnie Malienne de Textile (COMATEX-SA), SEGOU
}

\section{Assessing the physical working environments at the Malian Textile Company (COMATEX-SA), SEGOU}

\author{
Bagayoko TB ${ }^{*}$, Diakite $L^{2}$, Sanogo $\mathrm{S}^{3}$, Koumare $\mathrm{M}^{4}$, Sacko $\mathrm{I}^{5}$, Diallo $\mathrm{B}^{6}$
}

'Service de médecine légale/travail de Hôpital Nianankoro Fomba de Ségou (Mali)

${ }^{2}$ Centre de Santé de Référence Famory DOUMBIA (Ségou-Mali) 3Institut d'Etude et de Recherche en Géronto-gériatrie (Bamako-Mali) ${ }^{4}$ Service de médecine légale/travail de Hôpital du Point G (Mali) ${ }^{5}$ Centre National d'appareillage orthopédique du Mali

${ }^{6}$ Caisse Malienne de Sécurité Sociale (Bamako-Mali)

*Auteur correspondant : Dr Thierno Boubacar BAGAYOKO, Spécialiste en Santé et Sécurité au travail, chef de service de médecine légale/médecine de travail de Hôpital Nianankoro Fomba de Ségou (Mali), Tel : 00223793456 37, Email: thiernobag@gmail.com

\section{Résumé :}

Introduction: Les ambiances physiques au travail exposent aux risques professionnels spécifiques et pathologies associées. Elles peuvent affecter durablement la santé des salariés et compromettre la productivité de l'entreprise. Notre objectif était de comparer les facteurs d'ambiances physiques de travail à la COMATEX aux normes internationales en vigueur Méthodologie : il s'agissait d'une évaluation normative réalisée en 2015 à la COMATEX. Les facteurs d'ambiance au travail mesurés étaient : le bruit, la chaleur et l'hygrométrie, la lumière, la poussière de coton dans 122 postes de travail. Résultats : Les employés avaient moins de 40 ans dans $69 \%$ avec plus de cinq ans d'expérience de travail dans $56,07 \%$. Dans $67 \%$ des postes de travail, le bruit était excessif avec des extrêmes allant jusqu'à 105 décibels comparativement aux normes retenues par le Bureau international du travail qui sont inferieur à 85 décibels comme seuil d'alerte et 90 décibels comme seuil de danger. Le niveau de chaleur mesuré variait entre 26 et $52^{\circ} \mathrm{C}$ et l'humidité qui régnait dans les ateliers étaient inconfortables pour un travail lourd (seuil retenu pour un travail lourd est de $25^{\circ} \mathrm{C}$ et 26.7 pour un travail moyen et $30^{\circ} \mathrm{C}$ pour un travail léger, pour $29 \%$ d'humidité relative $\mathrm{RH}$ ). La quantité de poussière de coton dans l'air ambiant au niveau des postes de travail était de $1,4 \mathrm{mg} / \mathrm{cm}^{3}$ à $2,2 \mathrm{mg} / \mathrm{cm}^{3}$ (seuil inferieur $0.2 \mathrm{mg} / \mathrm{cm}^{3}$ ). La quantité de lumière fournie à chaque de poste de travail était entre 500 et 1000 lux. Aucun équipement de protection individuelle efficace n'était utilisé par les salariés de la COMATEX-SA. Conclusion : l'étude a révélé une énorme inconformité des ambiances physiques de travail mesurées à la COMATEX de Ségou. La nécessité d'appliquer la réglementation légale en santé et sécurité au travail est impérieuse dans cette entreprise, sinon au prix de la ruine des salariés.

Mots clés : Textile, Médecine de travail, COMATEX-SA, Ségou

\begin{abstract}
:
Introduction: The physical environments at work expose workers to specific occupational risks and its related pathologies. They can permanently affect the health of employees, and compromise the productivity of the company. Our objective was to compare the factors of physical working environments at COMATEX-SA with the current international standards. Methodology: This was a normative assessment carried out in 2015 at the COMATEX-SA. The factors measured in the work environment were: noise, heat and humidity, light, cotton dust in 122 workstations. Results: Employees were under 40 in $69 \%$ with more than five years of work experience in $56,07 \%$. In $67 \%$ of the workstations, the noise was excessive with extremes of up to 105 decibels compared to the standards adopted by the International Labor Office, which are below 85 decibels as a warning threshold and 90 decibels as a danger threshold. The measured heat level varied between 26 to $52^{\circ} \mathrm{C}$ and the humidity in the workshops was uncomfortable for heavy work (threshold selected for heavy work is $25^{\circ} \mathrm{C}$ and $26,7^{\circ} \mathrm{C}$ for average work and $30^{\circ} \mathrm{C}$ for light work, for $29 \%$ relative humidity $\mathrm{RH})$. The amount of cotton dust in the ambient air at the workstations was $1.4 \mathrm{mg} / \mathrm{cm}^{3}$ to $2.2 \mathrm{mg} / \mathrm{cm}^{3}$. (lower threshold $0,2 \mathrm{mg} / \mathrm{cm}^{3}$ ). The amount of light supplied to each workstation was between 500 and 1000 lux. No effective personal protective equipment was used by the employees at the COMATEX-SA.Conclusion: This study revealed an enormous discrepancy in the physical work environments measured at the COMATEX-SA. The need to apply legal regulations in occupational health and safety is imperative in this company, if not at the cost of the ruin of the employees.
\end{abstract}

Key words: Textile, health at work, COMATEX-SA, Ségou

\section{Introduction}

Créée en 1963 à Ségou au Mali, la Compagnie Malienne de Textile-SA (COMATEX-SA) représente la vitrine des industries manufacturières la plus ancienne du pays issue de l'indépendance. Elle est supposée avoir le système de santé et sécurité au travail le plus performant par rapport aux autres entreprises parce que jusque là gérée par les experts étrangers, avec un potentiel de 1500 salariés dont 904 ouvriers exposés aux risques professionnels divers. L'amélioration des conditions de travail et la santé des salariés passent par une bonne prévention des risques professionnels, l'application stricte des normes en vigueur en matière de Santé et Sécurité au Travail. Celles-ci se feront à travers des organes de suivi dynamiques et performants tels un comité d'hygiène, de sécurité et 
d'amélioration des conditions de travail. Un plaidoyer s'impose auprès des décideurs de l'entreprise, et soutenus par une bonne volonté politique.

La prévention des risques professionnels est une activité essentielle dans tout système de production, où le salarié doit être placé au centre des préoccupations. Cette activité s'exerce de façon transversale et verticale (prévention primaire, secondaire et tertiaire, etc...) au niveau de toutes les unités de production d'une industrie bien structurée $[1,2]$.

L'activité textile est une activité à haut risque mécanique, physique, ergonomique, biologique et environnemental. Les ambiances physiques qui règnent dans les ateliers de production (filature, tissage, teinture, énergies-eaux, etc...) constituent des risques professionnels non négligeables dont leurs contrôles obéissent à des seuils tolérables pour la santé des salariés [1,2]. Ces ambiances physiques composées de la chaleur, l'humidité, le bruit, la lumière et la poussière de coton peuvent, chacun en ce qui le concerne, affecter gravement la santé des salariés $[1,2]$. Une exposition prolongée à ces facteurs mérite une surveillance médicale particulière des salariés, et un contrôle régulier des conditions de travail [1-6].

Aucune mesure de ces facteurs d'ambiance ci-dessus citées, ni aucune étude n'avaient été faites dans cette industrie textile, pour déterminer leurs seuils, et cela pour des raisons techniques (la disponibilité des outils de mesure) ou administratives (la négligence des dirigeants de l'entreprise). Dans cette étude, notre objectif était de mesurer le niveau des facteurs d'ambiances physiques de travail à la COMATEX-SA de Ségou, et de les comparer aux normes internationales et de dégager le profil socioprofessionnel des salariés exposés.

\section{Méthodologie}

II s'agit d'une étude, normative réalisée et focalisée essentiellement sur l'atmosphère ambiante des postes de travail durant la période allant d'Octobre 2014 à Mai 2015. Certaines caractéristiques socioprofessionnelles ont été prises en compte par l'étude notamment la profession, l'âge, et l'ancienneté au poste. Notre étude s'est déroulée au sein de la COMATEX-SA de Ségou, la $4^{\mathrm{e}}$ région administrative du Mali, situé au centre du pays. Elle est une entreprise textile basée essentiellement sur la production du fil à tisser et du tissu. Elle emploie 1500 salariés dont 904 ouvriers et 596 autres cadres dans 122 postes de travail.Tous les postes de la chaine de production c'est-à-dire du cardage (lieu de départ du coton vrac, jusqu'au finissage écru, véritable laboratoire de vérification de la qualité du produit fini), l'administration, et les ateliers de mécanique et d'énergie-eaux ont fait l'objet de mesure des ambiances physiques de travail (le bruit, l'éclairage, la chaleur, l'humidité de l'air ambiant ou l'hygrométrie et la quantité de poussière de coton dans l'air respiré). Ces postes se répartissent entre les cinq départements essentiels de l'entreprise dont la filature, le tissage, le blanchiment, la teinture et l'impression, l'atelier de mécanique et énergie-eaux et les bureaux de l'administration.

Les instruments de mesure utilisés étaient le Sonomètre PYLE PSP01 pour mesurer le bruit, le thermomètre infrarouge TROTEC BP20 pour prendre la température ambiante du lieu, le luxmètre TROTEC BF05: pour mesurer l'intensité de la lumière, le thermo-hygromètre de type TROTEC BZ25 pour mesurer à la fois la température ambiante et l'humidité dans l'air, et l'appareil de mesure des poussières type DUST TRAK TSI pour mesurer la poussière de coton.

Les données relevées ont été comparées aux normes internationales retenues par le bureau international du travail (BIT):

\section{Analyse des données}

L'encodage et le traitement statistique des données ont été réalisés avec Excel version 8. L'étude descriptive des données a porté sur les postes de travail et un bref aperçu a porté sur certaines caractéristiques socioprofessionnelles des salariés travaillant dans les postes étudiées notamment leur âge, leur sexe, leur profession et leur ancienneté au poste. L'approche analytique a consisté à la description des résultats des mesures d'ambiance.

\section{Résultats}

Pour les salariés exposés à ces ambiances de travail, $60,27 \%$ étaient composés de la population ouvrière travaillant exclusivement sur les lignes de production. Et cette population d'étude était jeune avec $69 \%$ des âges de moins de 40 ans, et $56,07 \%$ parmi eux ont une ancienneté de plus de 05 ans dans l'entreprise (tableau II).

Au total, $67 \%$ des postes de travail étudiés $(n=122)$ présentaient une nocivité excessive du bruit pour les salariés, avec des extrêmes allant jusqu'à 105 décibels (tableau III).

La quantité de lumière fournie à chaque de poste de travail répondaient aux normes de l'Agence Française de Normalisation (AFNOR) soit comprise entre 500 et 1000 lux) sauf dans les postes de tissage où elle était restée très élevée, 1,5 fois de plus soit 1537 lux à la norme (tableau IV).

*La norme internationale en vigueur est de 300-1000 lux pour l'administration et les énergies-eaux et 500-1000 lux pour les autres ateliers.

Les ateliers de filature, de tissage, de blanchiment présentent un inconfort à la chaleur et à l'humidité avec des extrêmes allants de $26^{\circ} \mathrm{C}$ à $52^{\circ} \mathrm{C}$ pour un travail lourd (tableau V).

* La norme internationale en vigueur pour un travail lourd en textile est de $25^{\circ} \mathrm{C}$ (température) et $20-29 \mathrm{RH}$ (hygrométrie).

La quantité de poussière de coton relevée dans l'air ambiant par poste étudié était de $\left(2,2 \mathrm{mg} / \mathrm{cm}^{3}\right)$ au niveau de la filature et de $\left(1,4 \mathrm{mg} / \mathrm{cm}^{3}\right)$ au niveau du tissage (tableau VI).

*La norme internationale en vigueur pour un travail lourd en textile est de $0,2 \mathrm{mg} / \mathrm{cm}^{3}$. 
Au niveau des 122 postes de travail visités, aucun salarié ne disposait d'un équipement de protection individuelle efficace (EPI) contre la poussière de coton, le bruit, la forte luminosité et/ou la chaleur humide.

\section{Discussion}

Le bruit est le facteur d'ambiance physique en textile et le risque professionnel le plus important, le plus grave, et qui très vite ou durablement peut affecter la santé des salariés. II provient des machines utilisées, qui souvent pour la plupart les séries sont vielles [1-4]. Les valeurs extrêmes relevées dans notre étude oscillaient entre 70 à $105 \mathrm{~dB}$ pour $82 \%$ de nos postes de travail. Ce qui est supérieur aux normes internationales en matière de nuisances sonores. Nos résultats rejoignent plusieurs autres études effectuées en Afrique et les pays en voie de développement. E. TAH en Côte d'ivoire [1] et P.L KITRONZA [2] en Congo RDC trouvent dans leurs études respectives $88 \%$ de postes de travail émettant un bruit excessif. Aucun salarié de notre série $(0 \%)$ ne se protégeait contre le bruit, seuls $2,75 \%$ de l'effectif des salariés [1] dans la série de E. TAH se protégeaient contre le bruit, $85 \%$ des salariés respectivement aux Etats-Unis et en Allemagne [2]. L'enquête SUMER (surveillance médicale des expositions à haut risque professionnel) en France en 2010 trouve que $69,9 \%$ des salariés en textile portent un EPI avec plus de 20 heures d'exposition par semaine [3]. L'exposition prolongée et répétée aux nuisances sonores est responsable de surdité professionnelle qui est une surdité de perception par atteinte endocochléaire bilatérale, symétrique et irréversible avec stabilisation des lésions après cessation de l'exposition au bruit. Elle entraine également des effets extra auditifs, des effets neuropsychiques et cognitifs, des effets cardiovasculaires, des effets digestifs, des effets hormonaux [1-4].

Par rapport à l'éclairage dans notre entreprise textile, les ambiances lumineuses étaient les seules dans les normes AFNOR $[5,6]$ soit $66 \%$ des postes de travail étudiés. L'atelier de tissage lui seul présente un niveau de luminosité très fort par rapport au travail effectué $[5$, 6].Notre étude diffère de celle d'E. TAH [1] qui trouve que $22,8 \%$ des postes de travail mesurés de sa série respectent les normes en éclairage et de P.L KITRONZA [2] qui trouve que $18 \%$ de ses postes respectent les normes. Les ambiances lumineuses fortes ou faibles sont responsables de la fréquence élevée des accidents de travail et de troubles visuels $[5,6]$.

La chaleur et l'hygrométrie constituent séparément et conjointement deux ambiances chaudes et fréquentes au travail, et de façon transversale en textile même si elles coexistent avec le bruit et la poussière de coton dans les postes de la filature et du tissage [1-4]. .Aucun poste de travail de notre étude ne respecte les normes en matière de chaleur pour un travail lourd. Les températures effectives psychométriques mesurées allaient de $26^{\circ}$ à $32,9^{\circ} \mathrm{C}$ (Tableau V). Par contre P.L KITRONZA et al [2] trouvent que $6 \%$ des postes de travail dans sa série respectent les normes pour des mesures psychométriques allant de 24,5 à $35,5^{\circ} \mathrm{C}$. Egalement, l'enquête SUMER 2010, a montré que $30 \%$ des travailleurs en France sont confrontés à des nuisances thermiques élevées de $+30^{\circ} \mathrm{C}$ dont $10 \%$ dans le textile [3]. Une exposition prolongée à la chaleur affecte la santé des travailleurs et se manifeste par la déshydratation, les maux de tête, la fatigue, les crampes musculaires, l'insolation, le coup de chaleur, l'épuisement à la chaleur voire même la mort [7]. II convient cependant de noter que la lutte contre les températures élevées et l'humidité restent difficiles car ces dernières sont nécessaires pour certaines opérations, notamment en Filature [7]. Une humidité trop faible peut occasionner un inconfort en desséchant les membranes muqueuses, favoriser la présence de poussières dans l'air, contribuer aux éruptions cutanées [7]. Un taux d'humidité trop important dans l'air ambiant limite les possibilités de sudation, donc amplifie la sensation de chaleur. II rend également l'air du local lourd, et peut contribuer au développement de bactéries et moisissures [7].

La poussière de coton ne demeure pas moins importante comme nuisance en textile $[7,8]$. Les valeurs retrouvées dans notre étude dépassent tout commentaire, soit 1,4 à $2,2 \mathrm{mg} / \mathrm{cm}^{3}$ pour dix (10) postes de travail mesurés et pour huit (8) heures d'exposition en filature et tissage soit respectivement 7 à 11 fois supérieure à la norme. P.L KITRONZA et Al [2] et E TAH [1] n'en décrivent pas dans leurs études parmi les nuisances étudiées en textile. Les fibres textiles sont plus épaisses que les fibres d'amiante, de verre ou de céramique qui ont des diamètres inférieurs à $5 \mu \mathrm{m}$, et elles pénètrent moins profondément dans le poumon et y séjournent moins [8-10]. De ce point de vue, elles sont donc moins dangereuses, mais l'inhalation excessive de poussières textiles est néanmoins la cause de certaines maladies pulmonaires comme la bronchite chronique, la maladie pulmonaire obstructive chronique (MPOC), l'asthme et la byssinose, resserrement transitoire des voies respiratoires s'apparentant à l'asthme et donnant la «fièvre de filature» ou «fièvre du lundi» [810].

\section{Conclusion}

L'étude a révélé une inconformité très importante entre les normes prescrites en matière du bruit, de l'éclairage et de la chaleur en milieu de travail et la situation vécue par les salariés de la COMATEX-SA. De nouvelles recommandations portant sur le renforcement des mesures de prévention et l'amélioration des conditions de travail seront formulées. Loin d'être exhaustive, cette étude apparaît comme un plaidoyer à l'égard des autorités compétentes afin de prioriser la question du bien-être des employés de cette usine au travail. Elle ouvre par conséquent la voie à d'autres études plus approfondies dans ce domaine. 


\section{Références}

1.Tah $\mathrm{E}$. Etude des nuisances professionnelles dans une industrie textile, Mémoire des CES de santé au travail. Abidjan, 2006.

2.Kitronza PL, Philippe M. Environmental factors associated with textile industry in Democratic Republic of Congo: state of play.Pan Afr Med J. 2016 Sep 29;25:44. doi: 10.11604/pamj.2016.25.44.6479. eCollection 2016.

3.Amira S, Ast D. Des risques professionnels contrastes selon les metiers: Enquete SUMER 2010. References en sante au travail. 2014;140:29-54.

4.Rospide $P$, Descatha $A$. Recommandations à l'attention des équipes de santé au travail concernant la visite d'information et de prévention des salariés exposés au bruit: revue systématique. Archives des Maladies Professionnelles et de l'Environnement. 2019;80:404-414. 5. Association française de normalisation (AFNOR). Principes d'ergonomie visuelle applicables à l'éclairage des lieux de travail. Norme AFNOR X 35-103, 1980.
6. ALAN SMITH N. Les conditions d'éclairage général. Encyclopédie de sécurité et santé au travail. 3ème édition française volume 2; 46 :13-19.

7. Association française de normalisation (AFNOR), 1995: Exigences de sécurité pour le matériel textile, norme française homologuée NF EN ISO 11111 (Paris). 8.Lauwerys R, Haufroid V, Hoet P, Lison, D. Toxicologie industrielle et intoxications professionnelles. $5^{\mathrm{ème}}$ édition Issy-les-Moulineaux Cedex. Elsevier Masson SAS : Paris (2007)

9. American conference of governmental industrial hygienists. "Cotton dust, raw, untreated.» In: Documentation of the threshold limit values and biological exposure indices / Documentation of TLV's and BEl's. Cincinnati, Ohio : ACGIH. (2010). Publication \#0100Doc. http://www.acgih.org

10. Paris C. Syndrome toxic des poussières organiques «OrganicDusttoxic syndrome ODTS, référence en santé au travail N¹40 Décembre 2014

Liste des tableaux :

Tableau I: Normes internationales des ambiances physiques au travail recommandées par le Bureau International de Travail (BIT) pour huit (8) heures d'exposition.

\begin{tabular}{|l|c|}
\hline Nuisances étudiées & Normes recommandées \\
\hline \multirow{2}{*}{ Le bruit } & Seuil d'alerte : $85 \mathrm{~dB}(\mathrm{~A})$ \\
\cline { 2 - 2 } & Seuil de danger : $90 \mathrm{~dB}(\mathrm{~A})$ \\
\hline \multirow{2}{*}{ La lumière } & Travail lourd: $25^{\circ} \mathrm{C}$ \\
\hline Poussière de coton & Travail moyen : $26,7^{\circ} \mathrm{C}$ \\
\hline Plage « Humidex » pour & Travail léger : $30^{\circ} \mathrm{C}$ \\
\cline { 2 - 2 } l'hygrométrie dans l'air respiré [7]. & 500 à 1000 lux pour la lumière jaune \\
\hline $20-29 \mathrm{RH}$ & $0,2 \mathrm{mg} / \mathrm{cm}^{3}$ de poussières respirables ou ppm \\
\hline $30-39 \mathrm{RH}$ & Degré de confort \\
\hline $40-45 \mathrm{RH}$ & Sensation de bien-être : confortable \\
\hline $46 \mathrm{RH}$ et plus & Sensation de malaise \pm grande : \pm confortable \\
\hline
\end{tabular}

Tableau II: Caractéristiques socio-démographiques des employés de la COMATEX-SA

\begin{tabular}{|c|c|c|c|}
\hline \multicolumn{2}{|l|}{ Variables } & Fréquence & $\%$ \\
\hline \multirow[t]{2}{*}{ Sexe, $N=1500$} & Masculin & 1428 & 95.20 \\
\hline & Féminin & 72 & 4.80 \\
\hline \multirow{2}{*}{ Tranche d'âge, $\mathrm{N}=1500$} & $\leq 40$ ans & 1032 & 69 \\
\hline & $>40$ ans & 468 & 31 \\
\hline \multirow{2}{*}{ Catégories de travailleurs, $\mathrm{N}=1500$} & Ouvriers & 596 & 39.73 \\
\hline & Administrateurs & 904 & 60.27 \\
\hline \multirow{4}{*}{$\begin{array}{l}\text { Nombre d'années d'experience de travail } \\
\text { au poste actuel, } N=1500\end{array}$} & $0-5$ ans & 568 & 43.93 \\
\hline & $6-10$ ans & 217 & 16.78 \\
\hline & $11-15$ ans & 175 & 13.53 \\
\hline & $\geq 16$ ans & 333 & 25.75 \\
\hline
\end{tabular}


Tableau III : Niveau du bruit dans les ateliers de la COMATEX-SA apres après huit (8) heures d'exposition par salarié et par jour.

\begin{tabular}{|l|c|c|}
\hline Département de production & Niveau moyen du bruit* & Nombre de postes visités \\
\hline Filature & $95 \mathrm{~dB}$ & 40 \\
\hline Tissage & $105,3 \mathrm{~dB}$ & 40 \\
\hline Blanchiment, teinture, impression & $87 \mathrm{~dB}$ & 20 \\
\hline Administration & $52 \mathrm{~dB}$ & 12 \\
\hline Energies-eaux & $70 \mathrm{~dB}$ & 10 \\
\hline Total & & $\mathbf{1 2 2}$ \\
\hline
\end{tabular}

Tableau IV : Niveau d'éclairage à la COMATEX-SA après huit (8) heures d'exposition par salarié et par jour.

\begin{tabular}{|l|c|c|}
\hline Département de production & Eclairage moyen* & Postes visités \\
\hline Filature & 948 lux & 40 \\
\hline Tissage & 1537 lux & 20 \\
\hline Blanchiment, teinture, impression & 508 lux & 12 \\
\hline Administration et Autres & 310 lux & 10 \\
\hline Energies-eaux & 310 lux & 122 \\
\hline Total & & \\
\hline
\end{tabular}

Tableau V : Niveau de chaleur et d'humidité à la COMATEX-SA après huit (8) heures d'exposition par salarié et par jour.

\begin{tabular}{|l|c|c|c|}
\hline Département de production & $\begin{array}{c}\text { Température } \\
\text { moyenne* }\end{array}$ & $\begin{array}{c}\text { Hygrométrie } \\
\text { moyenne* }\end{array}$ & Postes visités \\
\hline Filature & 32,9 & 39,2 & 40 \\
\hline Tissage & 31,2 & 47,3 & 40 \\
\hline Blanchiment teinture impression & 26 & 51,45 & 20 \\
\hline Energie, eaux, & 30,7 & 32 & 10 \\
\hline Administration & 21 & 24 & 12 \\
\hline Total & & & 122 \\
\hline
\end{tabular}

Tableau VI :Quantité moyenne de poussière de coton dans l'air ambiant à la COMATEX-SA après huit (8) heures d'exposition par salarié et par jour.

\begin{tabular}{|l|c|c|}
\hline $\begin{array}{l}\text { Département } \\
\text { production }\end{array}$ & Quantité moyenne de poussière de coton dans l'air ambiant ${ }^{*}$ & $\begin{array}{c}\text { Nombre de points de } \\
\text { mesure }\end{array}$ \\
\hline Filature & 2.2 & 5 \\
\hline Tissage & 1,4 & 5 \\
\hline Total & & 10 \\
\hline
\end{tabular}

